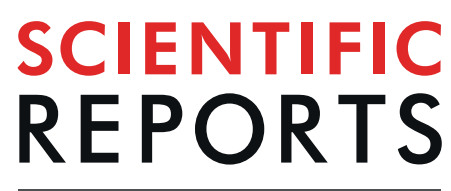

natureresearch

\title{
Normative data and associated factors of hand grip strength among elderly individuals: The Yilan Study, Taiwan
}

\author{
Po-Jung Pan ${ }^{1,2,8}$, Ching-Heng Lin ${ }^{1,3}$, Nan-Ping Yang ${ }^{1,4,8}$, Hsi-Chung Chen $\mathbb{1}^{5}$, \\ Hsuan-Ming Tsao ${ }^{1,6}$, Pesus Chou ${ }^{1}$ \& Nai-Wei Hsu ${ }^{1,6,7 *}$
}

Weak grip strength is associated with subsequent mortality in elderly populations. The normative data and associated factors of HGS in community-dwelling elderly Taiwanese populations require further evaluation. From February 2012 until the end of 2016, all residents of Yilan City, Taiwan aged 65 years or older were randomly selected for a population-based community health survey. A total of 2,470 older adults were enrolled in this study. The relationships between HGS and various anthropometric and sociodemographic correlates were examined. The results showed that HGS was higher in men than in women. The mean HGS exhibited a decreasing trend with advanced age in both men and women. HGS was significantly associated with height, weight, and exercise habits. The physical as well as the mental component summary measures of health-related quality of life (HROoL) were positively associated with HGS. After HROoL was integrated into the regression model, female sex, age, waist circumference, and diabetes mellitus were significantly negatively associated with HGS. In conclusion, HGS significantly decreased with advanced age. among community-dwelling Taiwanese elderly people, Various factors had different effects on HGS.

Hand grip and quadriceps muscle strength decrease with advanced age ${ }^{1}$. A cross-sectional analysis of 10,149 adults aged 60 years and older revealed that the prevalence of physical limitations, such as decrease of the activity of daily life (ADL) and the instrumental activity of daily life (IADL), was significantly higher among participants with low hand grip strength (HGS) than among those with high $\mathrm{HGS}^{2}$. Another recent systematic review of 21,197 participants demonstrated a relationship between low HGS and high hip fracture incidence in elderly people ${ }^{3}$. Moreover, a pooled analysis of 6,426 nondependent community-dwelling people aged 60 years and older revealed a $40 \%$ increase of the adjusted risk of all-cause mortality among elderly individuals with HGS in the lowest quartile; thus, weak grip strength was associated with subsequent mortality ${ }^{4}$. Therefore, HGS is a crucial indicator of health status and prognosis among elderly populations.

Normative data of grip strength across the life course were collected by Dodds et al. in Great Britain. After comparing these normative data with those of other world regions, they found that the grip strength measure obtained from people living in developing regions was clearly lower than that of people living in developed regions ${ }^{5}$. However, studies on HGS in Asia are few in number and were conducted later than those in Western countries. A 4-year longitudinal study recruited 3,018 community-dwelling Chinese individuals aged 64 years and older and found a more rapid decline in grip strength in women than in men. The study results also revealed that the grip strength in elderly people of Chinese descent was weaker than that in elderly people of African descent and those of European descent white people ${ }^{6}$. HGS among elderly people in Singapore was also lower

${ }^{1}$ Community Medicine Research Center \& Institute of Public Health, School of Medicine, National Yang-Ming University, Taipei, Taiwan. ${ }^{2}$ Department of Physical Medicine and Rehabilitation, National Yang-Ming University Hospital, Yilan, Taiwan. ${ }^{3}$ Department of Medical Research, Taichung Veterans General Hospital, Taichung, Taiwan. ${ }^{4}$ Department of Orthopedic Surgery, Hualien Hospital, Ministry of Health \& Welfare, Hualien, Taiwan. ${ }^{5}$ Department of Psychiatry \& Center of Sleep Disorders, National Taiwan University Hospital, Taipei, Taiwan. ${ }^{6}$ Division of Cardiology, Department of Internal Medicine, National Yang-Ming University Hospital, Yilan, Taiwan. ${ }^{7}$ Public Health Bureau, Yilan County, Taiwan. ${ }^{8}$ These authors contributed equally: Po-Jung Pan and Nan-Ping Yang. *email: nwhsu@ymuh. ym.edu.tw 
than that among elderly people in some Western countries ${ }^{7}$. Kim et al. demonstrated that among Koreans HGS peaked at 35-39 years of age and thereafter decreased in both sexes ${ }^{8}$. Additional studies are warranted, especially in other regions of Asia, to evaluate the normative data of HGS.

Age, sex, race/ethnicity, education, smoking status, body mass index, comorbidities, and physical activity have been associated with HGS in community-dwelling elderly people ${ }^{2}$. Greater height, higher weight, and smaller waist circumference have also been independently associated with higher HGS7 . Because HGS and its associated factors have become a major public health concern, especially among elderly populations worldwide, additional studies are required to thoroughly elucidate the various factors that influence HGS and the underlying associations. Therefore, the present study was designed to investigate the following: (1) the normative data of HGS and (2) the associated factors of HGS among community-dwelling elderly individuals in Taiwan.

\section{Methods}

Study design and participants. This study was part of the Yilan study series ${ }^{9-13}$, a population-based community health survey conducted by the Community Medicine Research Center of the National Yang-Ming University and the National Yang-Ming University Hospital in Taiwan. The study protocol was evaluated and approved by the Institutional Review Board (IRB) of National Yang-Ming University Hospital (IRB Approval No.: 2011A016). All the enrolled participants provided written informed consent. All methods were performed in accordance with the relevant guidelines and regulations.

The study methods have been described elsewhere ${ }^{9,11,14}$. In brief, from February 2012 to the end of 2016, all residents of Yilan City, located in the northeast of Taiwan, aged 65 years or older were randomly selected and invited to participate. Those who agreed to participate in the study were enrolled, and those who did not agree to participate were excluded. Additionally, those with preexisting hand problems were excluded from HGS measurement. Well-trained project assistants visited all the enrolled participants at their homes and conducted face-to-face interviews. Basic data and health-related information, including demographic characteristics, body weight/height, waist circumference, education level, living status (full-time living alone, part-time living alone, and not living alone), lifestyle (ie, smoking/drinking habits, exercise condition within the past 1 week, and sleep status), health-related quality of life (HRQoL) within the past 1 month, self-reported medical histories of chronic diseases (diabetes mellitus, hypertension, cardiovascular disease, hyperlipidemia, cerebrovascular accident, gout, and cataract) and current psychological status, and fall episodes within the past 1 year, were collected through physical examination and well-established questionnaires ${ }^{9,11,14}$. The Short-Form 12 Health Survey Version 2 (SF-12v2) was used for evaluating the HRQoL; the SF-12v2 comprises 12 items that constitute the mental component summary (MCS) and physical component summary (PCS) for measuring the participant's mental and physical function status in the past 4 weeks ${ }^{15,16}$.

Initially, 2,584 community-dwelling elderly individuals were randomly selected and interviewed. However, 114 of them were determined to have mild to moderate disability because of a previous cerebrovascular accident. Therefore, these participants were excluded from the present study. Finally, 2,470 subjects were enrolled.

Measurement of HGS. HGS of both hands was measured with a hydraulic hand dynamometer (Jamar, Jackson, MI, USA). Each participant completed the trial for each hand, and the final estimate of HGS was the average of all measurements ${ }^{10,17}$

Statistical analysis. Descriptive statistics are presented as numbers of cases, percentages, and means with standard deviation (SD). The independent $t$ test and the chi-squared test were used to analyze the differences in continuous and categorical variables, respectively, between groups. Box plots of HGS estimates were drawn, and their trend tests were evaluated using a simple linear regression. The beta $(\beta)$ coefficient and the partial R-squared of individual independent factors of HGS were calculated using stepwise multivariate linear regression to perform multivariate analyses.

\section{Results}

Sociodemographic data of participants. A total of 2,470 adults aged 65 years and older [998 (40.4\%) men and 1,472 (59.6\%) women] were enrolled in this study. A comparison of the basic characteristics, including age strata, chronic physical disorders, living status, and education level, of the sexes is presented in Table 1. The sex distribution, especially in the 65-69 years stratum, was significantly different. However, no significant difference in comorbidities such as heart disease (32.5\% vs $31.9 \%$ ) and diabetes mellitus (22.3\% vs $24.7 \%)$ was noted between men and women. Additionally, men exhibited lower rates of full-time and part-time living alone and higher rates of not living alone than women ( $5.4 \%$ vs $9 \% ; 2.4 \%$ vs $5.4 \%$; $92.2 \%$ vs $85.6 \%$, respectively). Furthermore, men also had higher education levels (junior high school or higher education level; $44.9 \%$ vs $23.0 \%)$.

Normative Data of HGS. Table 2 presents the means and SD of HGS by sex and age stratum. The mean values of HGS for men were significantly higher than those for women in sum $(25.4 \mathrm{~kg} v 15.5 \mathrm{~kg})$ or in subgroups by 2 - and 4-year age strata. The mean HGS dropped from $33.0 \mathrm{~kg}$ in men aged $65-69$ years to $21.1 \mathrm{~kg}$ in men aged 80 years or older. Moreover, HGS dropped from 19.1 to $12.6 \mathrm{~kg}$ in women. In the oldest stratum ( 75 years or older), the mean HGS values decreased to only 23.2 and $13.9 \mathrm{~kg}$ in men and women, respectively.

Minimum, maximum, median, and interquartile range of all HGS point estimates among various age strata for both sexes are presented in Figs. 1 and 2. Significantly decreasing trends $(\mathrm{p}<0.001)$ of HGS across all age strata were noted in both men and women. The medium values of HGS in men in all age strata were higher than those in women. 


\begin{tabular}{|c|c|c|c|c|c|c|c|}
\hline & \multicolumn{2}{|c|}{$\begin{array}{l}\text { Total } \\
(n=2470)\end{array}$} & \multicolumn{2}{|c|}{ Male } & \multicolumn{2}{|c|}{ Female } & \multirow{2}{*}{$\begin{array}{l}\text { p value of } \chi^{2} \\
\text { test }^{2}\end{array}$} \\
\hline & No. & $\%$ & No. & $\%$ & No. & $\%$ & \\
\hline \multicolumn{8}{|l|}{ Gender } \\
\hline male & 998 & 40.4 & $-\cdots-$ & $-\cdots-$ & $\mid-\cdots$ & 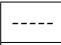 & \\
\hline female & 1472 & 59.6 & $-\cdots-$ & $-\cdots-$ & $\mid----$ & $-\cdots$ & \\
\hline \multicolumn{8}{|l|}{ Age Stratum (years) } \\
\hline $65-69$ & 270 & 10.9 & 61 & 6.1 & 209 & 14.2 & $<0.001$ \\
\hline $70-74$ & 711 & 28.8 & 269 & 27.0 & 442 & 30.0 & \\
\hline $75-79$ & 686 & 27.8 & 292 & 29.3 & 394 & 26.8 & \\
\hline 80 or more & 803 & 32.5 & 376 & 37.7 & 427 & 29.0 & \\
\hline \multicolumn{8}{|l|}{ Chronic Physical Disorders } \\
\hline Heart disease (yes) & 793 & 32.1 & 324 & 32.5 & 469 & 31.9 & 0.761 \\
\hline DM (yes) & 586 & 23.7 & 223 & 22.3 & 363 & 24.7 & 0.184 \\
\hline \multicolumn{8}{|l|}{ Living Status } \\
\hline full-time living alone & 186 & 7.5 & 54 & 5.4 & 132 & 9.0 & $<0.001$ \\
\hline part-rime living alone & 104 & 4.2 & 24 & 2.4 & 80 & 5.4 & \\
\hline not living alone & 2179 & 88.3 & 920 & 92.2 & 1259 & 85.6 & \\
\hline \multicolumn{8}{|l|}{ Educational Level } \\
\hline junior high school or higher & 785 & 31.8 & 447 & 44.9 & 338 & 23.0 & $<0.001$ \\
\hline none or primary school & 1681 & 68.2 & 548 & 55.1 & 1133 & 77.0 & \\
\hline
\end{tabular}

Table 1. Basic characteristics of the enrolled elderly participants. Abbreviations: DM: diabetes mellitus. Notes: ${ }^{\mathrm{a} C}$ Comparison between sexes.

\begin{tabular}{|c|c|c|c|c|c|c|c|c|c|c|}
\hline & \multicolumn{3}{|l|}{ Total } & \multicolumn{3}{|c|}{ Male } & \multicolumn{3}{|c|}{ Female } & \multirow{2}{*}{$\begin{array}{l}p \text { value of } \\
t \text { test }\end{array}$} \\
\hline & No. & mean & SD & No. & mean & SD & No. & mean & SD & \\
\hline In General (aged 65 years or more) & 2470 & 19.5 & 8.4 & 998 & 25.4 & 8.6 & 1472 & 15.5 & 5.4 & $<0.001$ \\
\hline \multicolumn{11}{|l|}{ Age Stratum (years) } \\
\hline \multicolumn{11}{|l|}{4 strata } \\
\hline $65-69$ & 270 & 22.3 & 7.9 & 61 & 33.0 & 6.8 & 209 & 19.1 & 4.8 & $<0.001$ \\
\hline $70-74$ & 711 & 21.5 & 8.6 & 269 & 29.0 & 7.9 & 442 & 16.9 & 5.1 & $<0.001$ \\
\hline $75-79$ & 686 & 19.8 & 8.2 & 292 & 25.9 & 7.8 & 394 & 15.3 & 4.7 & $<0.001$ \\
\hline 80 or more & 803 & 16.6 & 7.8 & 376 & 21.1 & 7.8 & 427 & 12.6 & 5.1 & $<0.001$ \\
\hline \multicolumn{11}{|l|}{2 strata } \\
\hline $65-74$ & 981 & 21.7 & 8.4 & 330 & 29.8 & 7.8 & 651 & 17.6 & 5.1 & $<0.001$ \\
\hline 75 or more & 1489 & 18.1 & 8.1 & 668 & 23.2 & 8.2 & 821 & 13.9 & 5.1 & $<0.001$ \\
\hline
\end{tabular}

Table 2. Means and standard deviations (SD) of hand grip strength (HGS; kg) by sex and age stratum. Notes: ${ }^{\mathrm{a}}$ Comparison between sexes.

Correlates of HGS. Stepwise multivariate linear regression analysis was conducted to evaluate the correlations of HGS with various factors. The results are presented in Table 3. For the overall sample, HGS was significantly positively associated with height $(\beta=0.19)$ and weight $(\beta=0.18)$ and slightly associated with exercise. Female sex, age, waist circumference, diabetes mellitus, and heart disease were independently associated with decreased HGS $(\beta=-7.5,-0.35,-0.12,-1.16$, and -0.63 , respectively). HGS was significantly higher in those living alone full time $(\beta=1.17)$. Stratified regression analysis in men and women indicated that HGS was positively associated with height $(\beta=0.24$ and 0.16$)$ and weight $(\beta=0.30$ and 0.13$)$ and negatively associated with age $(\beta=-0.53$ and -0.23$)$, waist circumstance $(\beta=-0.24$ and -0.09$)$ and diabetes mellitus $(\beta=-1.33$ and -0.10$)$ in both genders, and heart disease in women $(\beta=-0.64)$. The HGS was significantly stronger $(\beta=2.72)$ in men with full-time living alone status. For the overall sample, the highest R-squared percentage ( $36.4 \%$ in a total of $53.3 \%$ ) was noted for height. In men and women, the highest R-squared percentage values were obtained for age (20.9\% in a total of $35.8 \%$ and $16.1 \%$ in a total of $26.1 \%)$.

A stepwise multivariate linear regression analysis was performed again to evaluate the associated factors of HGS with the inclusion of HRQoL measured using the SF-12v2 with MCS and PCS. For the overall sample, HGS was significantly associated with height $(\beta=0.19)$, PCS $(\beta=0.19)$, MCS $(\beta=0.16)$, and weight $(\beta=0.15)$ and slightly associated with exercise after adjustment. Similarly, female sex, age, waist circumference, and diabetes mellitus were independently associated with decreased HGS $(\beta=-7.18,-0.31,-0.10$, and -0.93 , respectively). Stratified regression analysis in men and women demonstrated that HGS was positively associated with height $(\beta=0.24$ and 0.16$)$, PCS $(\beta=0.24$ and 0.14$)$, MCS $(\beta=0.21$ and 0.12$)$, and weight $(\beta=0.27$ and 0.11$)$ and negatively associated with age $(\beta=-0.47$ and -0.21$)$, waist circumstance $(\beta=-0.20$ and -0.07$)$, and diabetes 


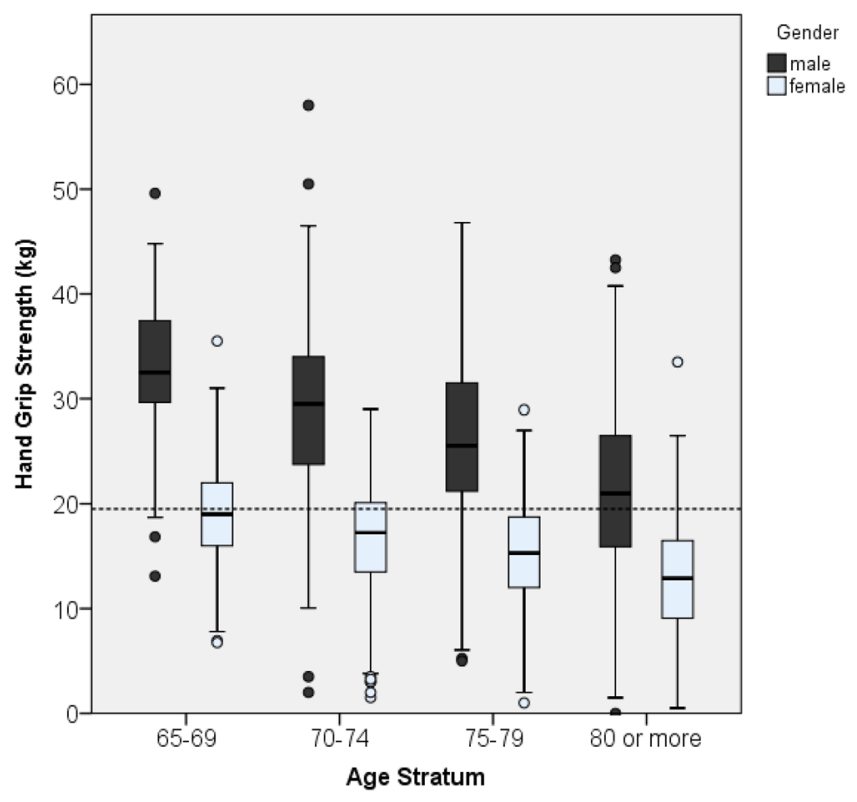

Figure 1. Box plots of HGS estimates in four age strata subgroups among men and women; trend test showing a $\mathrm{p}$ value of $<0.001$ for men and $<0.001$ for women.

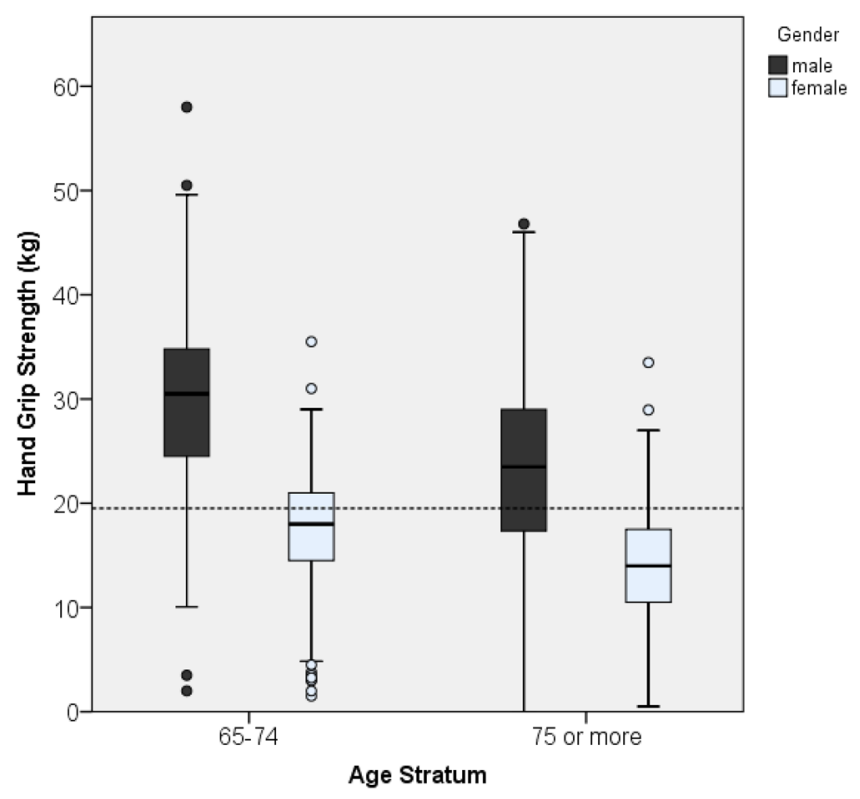

Figure 2. Box plots of HGS estimates in two age strata subgroups among men and women; trend test showing a $\mathrm{p}$ value of $<0.001$ for men and $<0.001$ for women.

mellitus $(\beta=-1.04$ and -0.08$)$. After controlling for height, age, PCS, MCS, weight, waist circumstance, and diabetes mellitus, men with full-time living alone status still had significantly stronger HGS $(\beta=2.35)$. For the overall sample, height had the highest R-squared percentage ( $36.4 \%$ in a total of $57.8 \%)$. In men and women, the highest R-squared percentage values were related to age (20.6\% in a total of $42.8 \%$ and $16.1 \%$ in a total of $33.1 \%)$.

\section{Discussion}

HGS is a crucial and easily available indicator for predicting the ability to perform daily functions, especially among elderly individuals ${ }^{18}$. In our study, 2,470 community-dwelling elderly persons were recruited and we conducted physical examination and face-to-face interviews at their homes ${ }^{11,14,17}$. Unlike the other studies in Taiwan, we used primary data collection with a large sample size ${ }^{5,19-21}$. Therefore, elderly individuals who previously tended to stay at home because of disability and could not go outside to undergo examinations could be assessed. Thus, even in the oldest age stratum ( 80 years or older), 803 residents were recruited in this study. 


\begin{tabular}{|c|c|c|c|c|c|c|c|c|c|}
\hline & \multicolumn{3}{|l|}{ Total $^{\mathbf{a}}$} & \multicolumn{3}{|l|}{ Male $^{\mathrm{b}}$} & \multicolumn{3}{|l|}{ Female $^{b}$} \\
\hline & $\begin{array}{l}\text { Beta }(\beta) \\
\text { coefficient }\end{array}$ & $\begin{array}{l}\text { Partial } \\
\text { R-square (\%) }\end{array}$ & Pvalue & $\begin{array}{l}\text { Beta }(\beta) \\
\text { coefficient }\end{array}$ & \begin{tabular}{|l|} 
Partial \\
R-square (\%)
\end{tabular} & Pvalue & $\begin{array}{l}\text { Beta }(\beta) \\
\text { coefficient }\end{array}$ & \begin{tabular}{|l} 
Partial \\
R-square (\%)
\end{tabular} & P value \\
\hline Height $(\mathrm{cm})$ & 0.19 & $36.4 \%$ & $<0.001$ & 0.26 & $9.8 \%$ & $<0.001$ & 0.15 & $5.7 \%$ & $<0.001$ \\
\hline $\begin{array}{l}\text { Gender (male as } \\
\text { reference) }\end{array}$ & -7.50 & $5.6 \%$ & $<0.001$ & & & & & & \\
\hline Age (years) & -0.35 & $8.7 \%$ & $<0.001$ & -0.53 & $20.9 \%$ & $<0.001$ & -0.23 & $16.1 \%$ & $<0.001$ \\
\hline Exercise (mins), in 1 week & 0.00 & $0.7 \%$ & $<0.001$ & 0.00 & $0.8 \%$ & 0.002 & 0.00 & $1.4 \%$ & $<0.001$ \\
\hline Weight (kgw) & 0.18 & $0.4 \%$ & $<0.001$ & 0.30 & $0.7 \%$ & $<0.001$ & 0.13 & $0.6 \%$ & $<0.001$ \\
\hline Waist circumference $(\mathrm{cm})$ & -0.12 & $0.9 \%$ & $<0.001$ & -0.24 & $2.6 \%$ & $<0.001$ & -0.09 & $1.3 \%$ & $<0.001$ \\
\hline DM (yes vs no) & -1.16 & $0.4 \%$ & $<0.001$ & -1.33 & $0.5 \%$ & 0.014 & -1.00 & $0.7 \%$ & 0.001 \\
\hline Heart disease (yes vs no) & -0.63 & $0.1 \%$ & 0.015 & & & & -0.64 & $0.3 \%$ & 0.016 \\
\hline \multicolumn{10}{|l|}{ Living Status } \\
\hline full-time living alone & 1.17 & $0.2 \%$ & 0.008 & 2.72 & $0.6 \%$ & 0.005 & & & \\
\hline part-time living alone & 0.46 & & 0.430 & -0.74 & & 0.606 & & & \\
\hline not living alone & & & & Reference & & & & & \\
\hline Model R-square & \multicolumn{3}{|l|}{$53.3 \%$} & \multicolumn{3}{|l|}{$35.8 \%$} & \multicolumn{3}{|l|}{$26.1 \%$} \\
\hline
\end{tabular}

Table 3. Correlations of HGS analyzed using stepwise multivariate linear regression. Abbreviation: DM: diabetes mellitus Notes: ${ }^{a}$ The model was adjusted using regression with stepwise selection for sex, age, heart disease, DM, living status, education level, height, weight, waist circumference, and exercise. ${ }^{\mathrm{b}}$ The model was adjusted using regression with stepwise selection for age, heart disease, DM, living status, education level, height, weight, waist circumference, and exercise.

Comparing the recently published normative data, we determined that the HGS of people aged 65 years or older in our study was lower than that evident in studies from European regions. For example, the mean values were approximately $13 \%$ lower than the results of the age-matched groups presented by Dodds et al. in $2014^{22}$. We also compared our results with the data obtained from other studies in Taiwan and determined that our mean values were similar to those reported by Wu et al. in $2009^{20}$, but slightly lower than the data reported by Liao et al. in $2014^{23}$. A comparison of our data with those reported in other Asian regions revealed that the mean values obtained in our study were lower than those obtained in the studies by Seino et al. in Japan and Kim et al. in $\mathrm{Korea}^{8,24}$, but were similar to those of another study reported by Ong et al. in Singapore in $2017^{7}$. It has been proposed that global variations in the norms of HGS were present, which might be attributable to the status of development ${ }^{5}$. Additionally, HGS has been determined to decrease with advanced age and among women ${ }^{1,4,5,22,25}$. After controlling for age and sex, several other factors have also been proposed to influence HGS, such as anthropometric elements (body weight, height, limb circumference, and palm length), testing protocol, study methods, measuring device, comorbidities, and other socioeconomic factors (ethnicity, education, marital status, and employment status) $5,7,19,23,24$. In our study, we proposed that the anthropometric differences might play a crucial role in the lower norms of HGS compared with the values reported for Caucasians in other studies because people in Taiwan have smaller physical stature. Additionally, it was reasonable to estimate that elderly individuals who accepted HGS measurement at certain community centers, similar to those in Liao's study, might have a more favorable health status because of their independent ambulation. By contrast, those who received interviews at home in our study might include those individuals with poor mobility and worse health status and with resultant lower HGS. This would indicate that the real norm of HGS in Taiwan might be lower than those reported in other studies.

HGS is a diagnostic criterion for sarcopenia, and the cutoff value in Asian populations has been proposed to be $<26 \mathrm{~kg}$ for men and $<18 \mathrm{~kg}$ for women. In our study, the cutoff points for both sexes were higher than the mean values $(25.4 \mathrm{~kg} \text { for men and } 15.5 \mathrm{~kg} \text { for women })^{26}$. As evident in Table 2, it is clear that men older than 75 years and women older than 70 years had mean HGS values below the threshold of sarcopenia. A higher percentage of elderly people in our study population might explain the lower HGS values obtained in our study.

In Figs. 1 and 2 illustrate the large gap between maximum and minimum HGS in each age stratum. Furthermore, the coefficient of variation increased with advanced age. We proposed that the increased variation in HGS with advanced age might be attributable to the accumulation of those correlation factors. Lino contended that various chronic diseases might be associated with low HGS, and thus in many elderly people, an increase in the number of comorbidities might lead to the large variation of $\mathrm{HGS}^{25}$.

In our study, age, sex, height, weight, hypertension, and diabetes were significantly associated with HGS, which was compatible with the results reported by Lino and Ramlagan ${ }^{25,27}$. As presented in Table 3, for each year increase of age after 65 years, an average reduction of $0.35 \mathrm{~kg}$ in HGS can be expected. A decrease of $3.5 \mathrm{~kg}$ in HGS for each decade of life can be estimated, which is substantially higher than that observed by Lino in Brazil ${ }^{25}$. Particularly, men exhibited a larger decline in HGS than women for each year of increase $(0.53 \mathrm{~kg} \mathrm{vs} 0.23 \mathrm{~kg})$. Therefore, prevention of muscle strength deterioration in elderly people, especially in men, should be a focus of interventions after retirement from their careers.

Although weight was positively associated with HGS, a negative association between waist circumference and HGS was also observed. Our result was consistent with the finding reported by Ong et al. in $2017^{7}$. This result suggested that older adults with a higher weight might have higher HGS; however, this did not hold true for obese 


\begin{tabular}{|c|c|c|c|c|c|c|c|c|c|}
\hline & \multicolumn{3}{|l|}{ Total $^{c}$} & \multicolumn{3}{|l|}{ Male $^{\mathrm{d}}$} & \multicolumn{3}{|l|}{ Female $^{d}$} \\
\hline & $\begin{array}{l}\text { Beta }(\beta) \\
\text { coefficient }\end{array}$ & \begin{tabular}{|l|} 
Partial \\
R-square (\%)
\end{tabular} & P value & $\begin{array}{l}\text { Beta }(\beta) \\
\text { coefficient }\end{array}$ & \begin{tabular}{|l|} 
Partial \\
R-square (\%)
\end{tabular} & P value & $\begin{array}{l}\text { Beta }(\beta) \\
\text { coefficient }\end{array}$ & \begin{tabular}{|l|} 
Partial R-square \\
$(\%)$
\end{tabular} & Pvalue \\
\hline Height $(\mathrm{cm})$ & 0.19 & $36.4 \%$ & $<0.001$ & 0.24 & $9.8 \%$ & $<0.001$ & 0.16 & $5.7 \%$ & $<0.001$ \\
\hline Gender (male as reference) & -7.18 & $5.6 \%$ & $<0.001$ & & & & & & \\
\hline Age (years) & -0.31 & $8.7 \%$ & $<0.001$ & -0.47 & $20.9 \%$ & $<0.001$ & -0.21 & $16.1 \%$ & $<0.001$ \\
\hline PCS score $^{\mathbf{a}}$ & 0.19 & $3.5 \%$ & $<0.001$ & 0.24 & $5.4 \%$ & $<0.001$ & 0.14 & $5.4 \%$ & $<0.001$ \\
\hline MCS score ${ }^{\mathbf{b}}$ & 0.16 & $2.2 \%$ & $<0.001$ & 0.21 & $3.4 \%$ & $<0.001$ & 0.12 & $3.9 \%$ & $<0.001$ \\
\hline Weight (kgw) & 0.15 & $0.4 \%$ & $<0.001$ & 0.27 & $0.7 \%$ & $<0.001$ & 0.11 & $0.4 \%$ & $<0.001$ \\
\hline Waist circumference $(\mathrm{cm})$ & -0.10 & $0.6 \%$ & $<0.001$ & -0.20 & $1.9 \%$ & $<0.001$ & -0.07 & $0.9 \%$ & $<0.001$ \\
\hline DM (yes vs no) & -0.93 & $0.2 \%$ & 0.001 & -1.04 & $0.3 \%$ & 0.044 & -0.80 & $0.4 \%$ & 0.004 \\
\hline Exercise (mins), in 1 week & 0.00 & $0.1 \%$ & 0.010 & & & & 0.00 & $0.3 \%$ & 0.008 \\
\hline \multicolumn{10}{|l|}{ Living Status } \\
\hline full-time living alone & & & & 2.35 & $0.5 \%$ & 0.010 & & & \\
\hline part-time living alone & & & & -0.94 & & 0.484 & & & \\
\hline not living alone & & & & Reference & & & & & \\
\hline Model R-square & \multicolumn{3}{|l|}{$57.8 \%$} & \multicolumn{3}{|l|}{$42.8 \%$} & \multicolumn{3}{|l|}{$33.1 \%$} \\
\hline
\end{tabular}

Table 4. Correlations of HGS analyzed using stepwise multivariate linear regression and including HRQoL

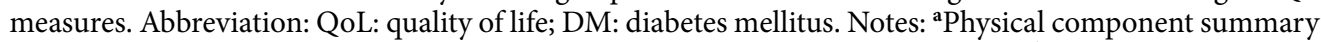
(PCS) measure of quality of life obtained using short-form (SF) - 12 questionnaire. ${ }^{\mathbf{b}}$ Mental component summary measure (MCS) of quality of life obtained using SF-12 questionnaire. ${ }^{c}$ The model was adjusted using regression with stepwise selection for sex, age, heart disease, DM, living status, education level, height, weight, waist circumference, exercise, PCS score, and MCS score. ${ }^{\mathrm{d}}$ The model was adjusted using regression with stepwise selection for age, heart disease, DM, living status, education level, height, weight, waist circumference, exercise, PCS score, and MCS score.

older adults. Elderly people with a higher body weight consisting of lean muscle rather than central fat had higher HGS.

Exercise may improve muscle strength in elderly people ${ }^{28}$. As presented in Table 3, exercise in the past 1 week was a significant factor associated with HGS but with a low beta coefficient. According to our records, most elderly individuals performed low- or low-to-moderate-intensity physical exercise such as walking, which was apparently not sufficient to improve HGS. Therefore, health-related specialists should design appropriate beneficial programs for promoting physical fitness improvement among elderly individuals.

Furthermore, also evident in Table 3, heart disease and full-time living alone status were significantly associated with HGS. However, after the introduction of HRQoL (Table 4), the association of HGS with both the factors disappeared and the total R-squared increased. HRQoL is an objective measure of the consequence of health status on quality of life. It measures the effects of various chronic illnesses, treatments, and disabilities on the physical and mental status experienced in daily life ${ }^{29}$. Therefore, the PCS of HRQoL had a positive association with HGS, and HRQoL might mediate the effects of heart disease and living status on HGS ${ }^{24}$. However, mental status has often been overlooked as one of the associated factors of HGS in elderly people. Depression has ever been observed to be negatively associated with $\mathrm{HGS}^{25,30}$. Pasco suggested that sarcopenia with resultant decreased strength and depression possibly shared a common pathophysiological pathway ${ }^{31}$. We have also previously demonstrated that depression is negatively associated with both MCS and PCS in elderly people ${ }^{11,14}$. Therefore, it would not be surprising for MCS to be positively associated with HGS.

Chronic diseases have been demonstrated to be associated with $\mathrm{HGS}^{12,13,15,25}$. In our study, both diabetes and heart disease were associated with HGS. Even with the introduction of HRQoL, diabetes exhibited a significantly negative association with HGS. Stenholm suggested that insulin resistance might have catabolic effects on muscle and resultant sarcopenia ${ }^{13}$. Mainous also suggested that sarcopenia and impaired metabolic states share common cellular and molecular characteristics ${ }^{12}$. These findings might partially explain the direct association between diabetes and HGS.

Our study analyzed the data from a population-based community health survey in Yilan City. For a more comprehensive survey, we have conducted a home-visit intervention. However, there were still some limitations in this study. First, some factors related to muscle strength, such as the nutritional status of the participants, were not included in this study. These factors may be included in future following-up studies with home-visit interventions $^{32}$. Second, for the HGS test, most of the older adults took the measurements in a standing position, with the exception of some older people who took the procedure in a sitting position to prevent falls. Although little difference was evident between these two test positions ${ }^{23}$, a more suitable sitting position should be applied to reduce the small intervention error in future research. Third, though the same two well-trained assistants could minimize the investigation bias, it took longer time for them to recruit these data from over 2,000 elderly individuals. Therefore, some changes of the community at macro level, such as the health policy of the government and the socioeconomic status of the inhabitants, might happen and affect these individuals' health outcomes during these 4 years. Forth, under the restriction of Personal Information Protection Act in Taiwan, we were not allowed to have the detailed names and addresses of these elderly individuals who were registered in Yilan city. Therefore, we could not but randomly selected and invited the old inhabitants to participate this study. It was not 
surprising that there were some differences when comparing the distribution of age and sex of these participants to that of the registered inhabitants in Yilan city ${ }^{33}$. This difference may limit the generalizability of our findings to the whole population in Taiwan. However, on the other hand, the elderly who agreed to participate our study might be the population who were prone to receive further community interventions. Therefore, the results of this study will provide us more sound data to plan and perform the following health promotion programs in the near future. Finally, some older adults did not participate in interviews for personal reasons, especially those in the age stratum of 65-69 years. Including more participants, especially in this age stratum, is necessary to obtain reliable results in the following-up studies. Despite these limitations, the results from this study provide important and valuable information for the government to arrange further steps to increase the health status among elderly people in the community.

\section{Conclusion}

Among community-dwelling Taiwanese elderly individuals, a significant decreasing trend of HGS with advanced age has been noted both in men and women. The HGS was independently positively associated with weight, height, PCS, MCS, and exercise habit and negatively associated with age, female sex, waist circumference, and a history of diabetes mellitus. Additionally, because this study recruited more elderly people with disabilities, the results suggested that the normative data of HGS in elderly Taiwanese people might be lower than those reported from previous studies.

\section{Data availability}

The datasets analyzed during the current study are available from the corresponding author on reasonable request.

Received: 21 May 2019; Accepted: 19 March 2020;

Published online: 20 April 2020

\section{References}

1. Samuel, D. et al. Age-associated changes in hand grip and quadriceps muscle strength ratios in healthy adults. Aging clinical and experimental research 24, 245-250 (2012).

2. Germain, C. M., Vasquez, E., Batsis, J. A. \& McQuoid, D. R. Sex, race and age differences in muscle strength and limitations in community dwelling older adults: Data from the Health and Retirement Survey (HRS). Arch Gerontol Geriatr 65, 98-103, https:// doi.org/10.1016/j.archger.2016.03.007 (2016).

3. Denk, K., Lennon, S., Gordon, S. \& Jaarsma, R. L. The association between decreased hand grip strength and hip fracture in older people: A systematic review. Experimental gerontology 111, 1-9, https://doi.org/10.1016/j.exger.2018.06.022 (2018).

4. Lera, L. et al. Reference values of hand-grip dynamometry and the relationship between low strength and mortality in older Chileans. Clinical interventions in aging 13,317-324, https://doi.org/10.2147/cia.S152946 (2018).

5. Dodds, R. M. et al. Global variation in grip strength: a systematic review and meta-analysis of normative data. Age Ageing 45 , 209-216, https://doi.org/10.1093/ageing/afv192 (2016).

6. Auyeung, T. W., Lee, S. W., Leung, J., Kwok, T. \& Woo, J. Age-associated decline of muscle mass, grip strength and gait speed: a 4-year longitudinal study of 3018 community-dwelling older Chinese. Geriatr Gerontol Int 14(Suppl 1), 76-84, https://doi.org/10.1111/ ggi.12213 (2014)

7. Ong, H. L. et al. Hand-grip strength among older adults in Singapore: a comparison with international norms and associative factors. BMC Geriatr 17, 176, https://doi.org/10.1186/s12877-017-0565-6 (2017).

8. Kim, C. R., Jeon, Y. J., Kim, M. C., Jeong, T. \& Koo, W. R. Reference values for hand grip strength in the South Korean population. PLoS One 13, e0195485, https://doi.org/10.1371/journal.pone.0195485 (2018).

9. Chen, H. C., Hsu, N. W. \& Chou, P. The association between extreme sleep duration and cardiac autonomic control in communitydwelling older adults: The Yilan Study. Taiwan. Journals of Gerontology. Series A: Biological Sciences and Medical Sciences 72, 929-936, https://doi.org/10.1093/gerona/glx045 (2017).

10. Chen, H. C., Hsu, N. W. \& Chou, P. The association between sleep duration and hand grip strength in community-dwelling older adults: The Yilan Study, Taiwan. Sleep 40, ZSX021, https://doi.org/10.1093/sleep/zsx021 (2017).

11. Hsu, N. W. et al. Different impacts of atrial fibrillation and cardiac premature contractions on the health-related quality of life in elderly people: The Yilan Study. Tohoku journal of experimental medicine 238, 75-83, https://doi.org/10.1620/tjem.238.75 (2016).

12. Mainous, A. G. 3rd, Tanner, R. J., Anton, S. D. \& Jo, A. Grip Strength as a Marker of Hypertension and Diabetes in Healthy Weight Adults. Am J Prev Med 49, 850-858, https://doi.org/10.1016/j.amepre.2015.05.025 (2015).

13. Stenholm, S. et al. Long-term determinants of muscle strength decline: prospective evidence from the 22-year mini-Finland followup survey. J Am Geriatr Soc 60, 77-85, https://doi.org/10.1111/j.1532-5415.2011.03779.x (2012).

14. Hsu, N. W., Tsao, H. M., Chen, H. C. \& Chou, P. Anxiety and depression mediate the health-related quality of life differently in patients with cardiovascular disease and stroke-preliminary report of the Yilan study: A population-based community health survey. PLoS ONE 9, e107609, https://doi.org/10.1371/journal.pone.0107609 (2014).

15. Maruish, M. E. \& Turner-Bowker, D. M. A guide to the development of certified modes of short form survey administration. Lincoln, RI: QualityMetric Incorporated (2009).

16. Maruish, M. E. \& DeRosa, M. A. A guide to the integration of certified Short Form survey scoring and data quality evaluation capabilities. Lincoln, RI: Quality Metric Incorporated (2009).

17. Yang, N. P. et al. Relationship between muscle strength and fall episodes among the elderly: the Yilan study, Taiwan. BMC Geriatr 18, 90, https://doi.org/10.1186/s12877-018-0779-2 (2018).

18. Leong, D. P. et al. Prognostic value of grip strength: findings from the Prospective Urban Rural Epidemiology (PURE) study. Lancet 386, 266-273, https://doi.org/10.1016/s0140-6736(14)62000-6 (2015).

19. Lee, M. C. et al. Criterion-Referenced Values of Grip Strength and Usual Gait Speed Using Instrumental Activities of Daily Living Disability as the Criterion. Journal of geriatric physical therapy (2001) 41, 14-19, https://doi.org/10.1519/jpt.0000000000000106 (2018).

20. Wu, S. W., Wu, S. F., Liang, H. W., Wu, Z. T. \& Huang, S. Measuring factors affecting grip strength in a Taiwan Chinese population and a comparison with consolidated norms. Applied ergonomics 40, 811-815, https://doi.org/10.1016/j.apergo.2008.08.006 (2009).

21. Mat Jais, I. S., Chan, K. L., Loke, M. K. A., Abdul Rahim, S. \& Tay, S. C. Normative data on functional grip strength of elderly in Singapore. Journal of hand therapy: official journal of the American Society of Hand Therapists 31, 122-128, https://doi.org/10.1016/j. jht.2016.10.004 (2018). 
22. Dodds, R. M. et al. Grip strength across the life course: normative data from twelve British studies. PLoS One 9, e113637, https://doi. org/10.1371/journal.pone.0113637 (2014).

23. Liao, W. C., Wang, C. H., Yu, S. Y., Chen, L. Y. \& Wang, C. Y. Grip strength measurement in older adults in Taiwan: a comparison of three testing positions. Australasian journal on ageing 33, 278-282, https://doi.org/10.1111/ajag.12084 (2014).

24. Seino, S. et al. Reference values and age and sex differences in physical performance measures for community-dwelling older Japanese: a pooled analysis of six cohort studies. PLoS One 9, e99487, https://doi.org/10.1371/journal.pone.0099487 (2014).

25. Lino, V. T. et al. Handgrip Strength and Factors Associated in Poor Elderly Assisted at a Primary Care Unit in Rio de Janeiro, Brazil. PLoS One 11, e0166373, https://doi.org/10.1371/journal.pone.0166373 (2016).

26. Chen, L. K. et al. Sarcopenia in Asia: consensus report of the Asian Working Group for Sarcopenia. Journal of the American Medical Directors Association 15, 95-101, https://doi.org/10.1016/j.jamda.2013.11.025 (2014).

27. Ramlagan, S., Peltzer, K. \& Phaswana-Mafuya, N. Hand grip strength and associated factors in non-institutionalised men and women 50 years and older in South Africa. BMC research notes 7, 8, https://doi.org/10.1186/1756-0500-7-8 (2014).

28. Mieszkowski, J. et al. The Effect of Nordic Walking Training Combined with Vitamin D Supplementation on Postural Control and Muscle Strength in Elderly People-A Randomized Controlled Trial. Int J Environ Res Public Health 15, https://doi.org/10.3390/ ijerph15091951 (2018).

29. Office of Disease Prevention and Health Promotion. Healthy People 2020: Foundation Health Measure Report Health-Related Quality of Life and Well-Being., https://www.healthypeople.gov/2020/about/foundation- health-measures/ Health-Related-Quality-of-Lifeand-Well-Being (2010).

30. Brooks, J. M. et al. Depression and Handgrip Strength Among USAdults Aged 60 Years and Older from NHANES 2011-2014. J Nutr Health Aging 22, 938-943, https://doi.org/10.1007/s12603-018-1041-5 (2018).

31. Pasco, J. A. et al. Sarcopenia and the Common Mental Disorders: a Potential Regulatory Role of Skeletal Muscle on Brain Function? Current osteoporosis reports 13, 351-357, https://doi.org/10.1007/s11914-015-0279-7 (2015).

32. Mendes, J. et al. Association of Anthropometric and Nutrition Status Indicators with Hand Grip Strength and Gait Speed in Older Adults. JPEN. Journal of parenteral and enteral nutrition 43, 347-356, https://doi.org/10.1002/jpen.1424 (2019).

33. Lai, H. C., Hsu, N. W., Chou, P. \& Chen, H. C. The Associations between Various Sleep-wake Disturbances and Depression in Community-dwelling Older Adults- the Yilan Study, Taiwan. Aging Ment Health, https://doi.org/10.1080/13607863.2019.1582006 (2019).

\section{Acknowledgements}

The authors would like to thank the Community Angels (social volunteers) and the Yang-Ming Crusade (student volunteers of the National Yang-Ming University) for their help in the community survey. The present study was partially supported financially by National Yang-Ming University Hospital, Taichung Veterans General Hospital (TCVGH-YM1070203), and the Ministry of Science and Technology (MOST: 107-2314-B-010-049). The National Yang-Ming University Hospital, Taichung Veterans General Hospital, and the Ministry of Science and Technology had no role in the study design, data collection, analysis, interpretation, and writing the manuscript.

\section{Author contributions}

The study was designed by P.C. and N.W.H.; data were gathered and analyzed by N.W.H., H.M.T., P.J.P., N.P.Y., and C.H.L.; the initial draft of the manuscript was written by P.J.P., N.P.Y., N.W.H., and C.H.L.; the accuracy of the data and analyses was assured by H.C.C., N.W.H., and P.C. All authors participated in the preparation of the manuscript and approved the final version. All authors have read and approved the final manuscript. In general, P.J.P. and N.P.Y. contributed equally to the work.

\section{Competing interests}

The authors declare no competing interests.

\section{Additional information}

Correspondence and requests for materials should be addressed to N.-W.H.

Reprints and permissions information is available at www.nature.com/reprints.

Publisher's note Springer Nature remains neutral with regard to jurisdictional claims in published maps and institutional affiliations.

(c) (1) Open Access This article is licensed under a Creative Commons Attribution 4.0 International License, which permits use, sharing, adaptation, distribution and reproduction in any medium or format, as long as you give appropriate credit to the original author(s) and the source, provide a link to the Creative Commons license, and indicate if changes were made. The images or other third party material in this article are included in the article's Creative Commons license, unless indicated otherwise in a credit line to the material. If material is not included in the article's Creative Commons license and your intended use is not permitted by statutory regulation or exceeds the permitted use, you will need to obtain permission directly from the copyright holder. To view a copy of this license, visit http://creativecommons.org/licenses/by/4.0/.

(C) The Author(s) 2020 